\title{
Insights and relative effect of aspirin, naproxen and ibuprofen containing hydrogels: From design to performance as a functional dual capacity restorative material and build in free radical defense: In-vitro studies
}

\author{
Victoria Tamara Perchyonok ${ }^{1}$, Vanessa Reher ${ }^{1}$, Shengmiao Zhang ${ }^{2}$, Sias R. Grobler ${ }^{3}$, \\ Theunis G. Oberholzer ${ }^{1}$, Ward Massey ${ }^{1}$ \\ ${ }^{1}$ School of Dentistry and Oral Health, Griffith University, Southport, Australia \\ ${ }^{2}$ School of Material Science and Engineering, East China University of Science and Technology, Shanghai, China \\ ${ }^{3}$ Oral and Dental Research Institute, Faculty of Dentistry, University of the Western Cape, Cape Town, South Africa \\ Email: tamaraperchyonok@gmail.com
}

Received 9 January 2014; revised 13 February 2014; accepted 20 February 2014

Copyright (C 2014 Victoria Tamara Perchyonok et al. This is an open access article distributed under the Creative Commons Attribution License, which permits unrestricted use, distribution, and reproduction in any medium, provided the original work is properly cited. In accordance of the Creative Commons Attribution License all Copyrights (C) 2014 are reserved for SCIRP and the owner of the intellectual property Victoria Tamara Perchyonok et al. All Copyright @ 2014 are guarded by law and by SCIRP as a guardian.

\section{ABSTRACT}

Restorative materials in the new era aim to be "bioactive" and long-lasting. It has been suggested that the anti-inflammatory activity of some non-steroidal anti-inflammatory drugs (NSAIDs) may be partly due to their ability to scavenge reactive oxygen species (ROS) and reactive nitrogen species (RNS), as well as to inhibit the respiratory burst of neutrophils triggered by various activating agents. As a part of our continuous interest of developing functional dual action restorative materials capable of being "bio-active" and long-lasting, we design and evaluate novel chitosan hydrogels containing krill oil (antioxidant containing material), naproxen, ibuprofen (non steroidal anti-inflammatory medication), aspirin (pain relieve medication and free radical scavengers) and combinations thereof (chitosan-H-krill oil, chitosan-H-krill oil-aspirin and chitosan-H-naproxen, chitosan-H-naproxen-krill oil, chitosan-H-krill oil-ibuprofen and chitosan-H-ibuprofen) as functional additive prototypes for further development of "dual function restorative materials"; secondly, determine their effect on the dentin bond strength of a composite and thirdly, evaluate the capability of newly designed hydrogels to play an integral role of "build in" free radical defense mechanism by using BSA solubility as a "molecular prototype" of the site of free radical attack in vitro. Materials and Methods: The above mentioned hydrogels were prepared by dispersion of the corresponding component in glycerol and acetic acid with the addition of chitosan gelling agent. The surface morphology (SEM), release behaviors (physiological pH and also in acidic conditions), stability of the therapeutic agent-antioxidant-chitosan and the effect of the hydrogels on the shear bond strength of dentin were also evaluated. Results: The release of aspirin, ibuprofen and naproxen confers the added benefit of synergistic action of a functional therapeutic delivery when comparing the newly designed chitosan-based hydrogel restorative materials to the commercially available products alone. Neither the release of aspirin, ibuprofen or naproxen nor the antioxidant stability was affected by storage over a 6month period. The hydrogel formulations have a uniform distribution of drug content, homogenous texture and yellow color (SEM study). All chitosan dentin treated hydrogels gave significantly $(P<0.05$; non-parametric ANOVA test) higher shear bond values $(P<0.05)$ than dentin treated or not treated with phosphoric acid. The model protein (BSA) was adopted to evaluate the chitosan-based functional biomaterials as defense for undesired free radical formation under in vitro conditions. Conclusion: The added benefits of the chitosan treated hydrogels involved positive influence on the aspirin, ibuprofen and naproxen release, increased dentin bond strength as well as demonstrated in vitro "build in" free radical defense mechanism, therefore acting as a "proof of concept" for the functional multi-dimentional restorative materials with the build in free radical defense me- 


\section{chanism.}

\section{KEYWORDS}

\section{Therapeutic Polymers; Adhesives; Chitosan; Hydrogels; Asprin; Ibuprofen; Naproxen; Dentin Bonding; Antioxidants; Bioactive}

\section{INTRODUCTION}

Facial arthromyalgia (FAM) or the temporomandibular joint pain dysfunction syndrome is a common condition in which patients complain of pain and tenderness in one or both temporomandibular joints (TMJ), often with limitation of jaw opening [1]. However, the precise underlying biochemical mechanisms leading to both pain and joint dysfunction remain to be established [1-3]. It has been suggested that the anti-inflammatory activity of some non-steroidal anti-inflammatory drugs (NSAIDs) may be partly due to their ability to scavenge reactive oxygen species (ROS) and reactive nitrogen species (RNS), as well as to inhibit the respiratory burst of neutrophils triggered by various activating agents [4]. Hydrogels are hydrophilic three-dimensional polymer networks, which contain a large amount of water [5]. They are highly permeable to various drugs and the entrapped drugs can be released through their web-like structures [6].

\section{Null Hypothesis}

As a part of our continuous interest of developing functional dual action restorative materials capable of being "bio-active" and long-lasting, we designed and evaluate novel chitosan hydrogels containing krill oil (antioxidant containing material), naproxen, ibuprofen (non steroidal anti-inflammatory medication), aspirin (pain relieve medication and free radical scavengers) and combinations thereof (chitosan-H-krill oil, chitosan-H-krill oil-aspirin and chitosan-H-naproxen, chitosan-H-naproxen-krill oil, chitosan-H-krill oil-ibuprofen and chitosan-H-ibuprofen) as functional additive prototypes for further development of "dual function restorative materials," secondly to determine their effect on the dentin bond strength of a composite and thirdly evaluate the capability of newly designed hydrogels to play an integral role of "build in free radical defense mechanism by using BSA solubility as a” molecular prototype of the site of free radical attack in vitro.

\section{MATERIALS AND METHODS}

Chitosan (Aldrich, Australia), glycerol (Sigma, USA), glacial acetic acid (E. Merck, Germany) were used as received. The degree of de-acetylation of typical commercial chitosan used in this study is $87 \%$. Chitosan with molecular weight $2.5 \times 10^{3} \mathrm{KD}$ was used in the study. The isoelectric point is 4.0 - 5.0. Krill Oil (Aurora Pharmaceuticals, Australia), Aspirin (Safeway, Australia), Ibuprofen (Safeway, Australia) and Naproxen (Safeway, Australia) were used as received.

\subsection{Preparation of Various Naproxen: Additive Hydrogels Gels}

The naproxen containing gel was prepared by dispersion of naproxen powder $0.2 \mathrm{gm}$ in glycerol $(5 \% \mathrm{w} / \mathrm{w})$ using a mortar and a pestle. Ten milliliters of glacial acetic acid (3\% w/w) was then added with continuous mixing and finally chitosan polymer was spread on the surface of the dispersion and mixed well to form the required gel. The strength of the prepared gel (10 gm) is $0.3 \mathrm{~g}$ naproxen in each gm of the base. Naproxen gel had been prepared with three different concentrations of chitosan gelling agent $(5 \% \mathrm{w} / \mathrm{w})$. The summary of the newly prepared materials was presented in Table 1.

Table 1. Gel formulation prepared in the study.

\begin{tabular}{|c|c|c|c|c|c|c|c|}
\hline Gel formulation & & Chitosan (w/w\%) & Aspirin (w/w\%) & Krill Oil (w/w\%) & Ibuprofen & Naproxen & $\mathbf{p H}$ \\
\hline Chitosan-H & Gel-1 & 5 & 0 & 0 & 0 & 0 & 5.60 \\
\hline Chitosan-H-A1 & Gel-2 & 5 & 1 & 0 & 0 & 0 & 5.92 \\
\hline Chitosan-H-A1K & Gel-3 & 5 & 1 & 1 & 0 & 0 & 6.10 \\
\hline Chitosan-H-N1 & Gel-4 & 5 & 0 & 0 & 0 & 1 & 5.87 \\
\hline Chitosan-H-N1K1 & Gel-5 & 5 & 0 & 1 & 0 & 1 & 5.90 \\
\hline Chitosan-H-I1 & Gel-6 & 5 & 0 & 0 & 1 & 0 & 5.96 \\
\hline Chitosan-H-I1K1 & Gel-7 & 5 & 0 & 0 & 1 & 1 & 6.10 \\
\hline
\end{tabular}

Where $\mathrm{K}$ is Krill Oil additive, A is aspirin additive, $\mathrm{N}$ is the naproxen, I-ibuprofen. Hydrogels containing chitosan (5\%) are synthesized and characterized. 


\subsection{Preparation of Various Aspirin: Additives Hydrogels Gels}

The aspirin containing gel was prepared by dispersion of aspirin powder $0.3 \mathrm{gm}$ in glycerol (5\% w/w) using a mortar and a pestle following the methodology described for naproxen containing hydrogels. The summary of the newly prepared materials is highlighted in Table 1.

\subsection{Preparation of Various Ibuprofen: Additive Hydrogels Gels}

The ibuprofen containing gel was prepared by dispersion of naproxen powder $0.2 \mathrm{gm}$ in glycerol $(5 \% \mathrm{w} / \mathrm{w})$ using a mortar and a pestle following the methodology described for naproxen containing hydrogels. The summary of the newly prepared materials is highlighted in Table 1.

Where $\mathrm{K}$ is Krill Oil additive, $\mathrm{A}$ is aspirin additive, $\mathrm{N}$ is the naproxen, I-ibuprofen. Hydrogels containing chitosan (5\%) are synthesized and characterized.

\subsection{Determination of Gel pH}

One gram of the prepared gels was accurately weighed and dispersed in $10 \mathrm{ml}$ of purified water. The $\mathrm{pH}$ of the dispersions was measured using a $\mathrm{pH}$ meter (HANNA instruments, HI8417, Portugal).

\subsection{In Vitro Study of Aspirin Release Profile}

The release study was carried out with USP dissolution apparatus type 1 , Copley UK, slightly modified in order to overcome the small volume of the dissolution medium, by using $100 \mathrm{ml}$ beakers instead of the jars. The basket of the dissolution apparatus $(2.5 \mathrm{~cm}$ in diameter) was filled with $1 \mathrm{gm}$ of asprin gel on a filter paper. The basket was immersed to about $1 \mathrm{~cm}$ of its surface in 50 $\mathrm{ml}$ of phosphate buffer $\mathrm{pH} 6.8$, at $37^{\circ} \mathrm{C} \pm 0.5^{\circ} \mathrm{C}$ and 100 rpm [7]. Samples (2 ml) were collected at 0.25, 1, 2, 3, 4, 5, 6, 7 and 8 hours [8] and were analyzed spectrophotometrically by U.V. Spectrophotometer (Cintra 5, GBC Scientific equipment, Australia) at $\lambda \max 275 \mathrm{~nm}$ [9]. Each sample was replaced by the same volume of phosphate buffer $\mathrm{pH} 6.8$ to maintain its constant volume and sink condition [10].

\subsection{Evaluation of in Vitro Ibuprofen Release}

The release study was carried out with USP dissolution apparatus type 1, Copley UK, slightly modified in order to overcome the small volume of the dissolution medium, by using $100 \mathrm{ml}$ beakers instead of the jars. The basket of the dissolution apparatus $(2.5 \mathrm{~cm}$ in diameter) was filled with $1 \mathrm{gm}$ of ibuprofen gel on a filter paper. The basket was immersed to about $1 \mathrm{~cm}$ of its surface in 50 $\mathrm{ml}$ of phosphate buffer $\mathrm{pH} 6.8$, at $37^{\circ} \mathrm{C} \pm 0.5^{\circ} \mathrm{C}$ and 100 rpm [7]. Samples (2 ml) were collected at 0.25, 1, 2, 3, 4,
5, 6, 7 and 8 hours $[7,8]$ and were analyzed spectrophotometrically by U.V. Spectrophotometer (Cintra 5, GBC Scientific equipment, Australia) at $\lambda \max 264 \mathrm{~nm} \mathrm{~nm}$ [9]. Each sample was replaced by the same volume of phosphate buffer $\mathrm{pH} 6.8$ to maintain its constant volume and sink condition [10].

\subsection{In-Vitro Drug Naproxen Release}

The release study was carried out with USP dissolution apparatus type 1, Copley U.K., slightly modified in order to overcome the small volume of the dissolution medium, by using $100 \mathrm{ml}$ beakers instead of the jars. The basket of the dissolution apparatus (2.5 cm in diameter) was filled with $1 \mathrm{gm}$ of naproxen gel on a filter paper. The basket was immersed to about $1 \mathrm{~cm}$ of its surface in 50 $\mathrm{ml}$ of phosphate buffer $\mathrm{pH} 6.8$, at $37^{\circ} \mathrm{C} \pm 0.5^{\circ} \mathrm{C}$ and 100 rpm [7]. Samples (2 ml) were collected at $0.25,1,2,3,4$, 5, 6, 7 and 8 hours [8] and were analyzed spectrophotometrically by U.V. Spectrophotometer (Cintra 5, GBC Scientific equipment, Australia) at $\lambda \max 260 \mathrm{~nm}$ [9]. Each sample was replaced by the same volume of phosphate buffer $\mathrm{pH} 6.8$ to maintain its constant volume and sink condition [10].

\subsection{Morphology of the Gels}

The samples were prepared by freezing in liquid nitrogen for $10 \mathrm{~min}$, and then were freeze-dried for $24 \mathrm{~h}$. The prepared samples were fractured in liquid nitrogen using a razor blade. The fractured samples were attached to metal stubs, and sputter coated with gold under vacuum for SEM. The interior and the surface morphology were observed in scanning electron microscope (SEM, Hitachi S4800, Japan).

\subsection{Gel Stability}

Stability of the gel formulations was also investigated. The organoleptic properties (color, odor), $\mathrm{pH}$, drug content, and release profiles of the gels stored at $20^{\circ} \mathrm{C}$ were examined on days $(0,15,30$ and 178$)$.

\subsection{Studies of Equilibrium Swelling in the Alternative Drug Delivery Systems}

A known weight functionalized chitosan containing dry gels were immersed in $\mathrm{pH} 4.0, \mathrm{pH} 9.0$ buffer solutions, respectively, and kept at $25^{\circ} \mathrm{C}$ for $48 \mathrm{~h}$ until equilibrium of swelling had been reached. The swollen gels were taken out and immediately weighed with microbalance after the excess of water lying on the surfaces was absorbed with a filter paper. The equilibrium swelling ratio (SR) was calculated using the following equation:

$$
S R=\left(W_{s}-W_{d}\right) / W_{d} \times 100 \%
$$


where $W_{s}$ and $W_{d}$ are the weights of the gels at the equilibrium swelling state and at the dry state, respectively [11]. Experiments were repeated in triplicate for each gel specimen and the mean value calculated.

\subsection{Shear Bond Strength Tests for Dentin Bonding}

Extracted non-carious, intact, human molars stored in water containing a few crystals of thymol at $4^{\circ} \mathrm{C}$ were used within two months. Samples were checked before use for any damage caused by their removal. The roots of the teeth were removed with a separating disc and the occlusal enamel removed by grounding wet on 60-grit silicon carbide $(\mathrm{SiC})$ paper. The teeth were embedded in PVC (Consjit Tubing, SA PVC, JHB, RSA) pipe containers with cold cure acrylic resin so that the grounded occlusal surfaces projected well above the resin. The 10 $\mathrm{mm}$ length pipes were put on a glass surface with one end blocked by the glass and the embedding done through the open end. Immediately after embedding the occlusal surfaces were ground wet with 180-grit followed by 600 -grit $\mathrm{SiC}$ on a polishing machine to expose the superficial dentin. The samples were washed under a stream of tap water. A standardized zig (Ultradent ISO A2-70) with an internal diameter of $2.5 \mathrm{~mm}$ and height of $3 \mathrm{~mm}$ was used to shape the composite resin stud (SDR, Dentsply, CA, USA, Batch number 1105000609, Exp 2013-04). Two of these studs were then bonded to the polished dentin surface of each tooth via the bonding agent XP bond (Dentsply, New York, USA), as suggested by the manufacturer. The bonding agent contained: carboxylic acid modified dimethacrylate (TCB resin), phosphoric acid modified acrylate resin (PENTA), urethane dimetacrylate (UDMA), triethyleneglycol dimethacrylate (TEGDMA), 2-hydroxyethylmethacrylate (HEMA), butylated benzenediol (stabilizer), ethyl-4-dimethylaminobenzoate), camphorquinone, functionalized amorphous silica, t-butanol.

In this way were 80 teeth samples (each containing 2 studs) prepared and divided into 10 groups of 8 each, A$\mathrm{N}$ (Table 2) and stored in a solution of artificial saliva. These groups were then treated as outlined in Table 2. After 24 hours one stud of each tooth was tested for shear bond strength and the other one after 3 months. An Instron Universal Testing Machine (Griffith University, G12) at a crosshead speed of $0.5 \mathrm{~mm} /$ minute was used to test the de-bonding strength. All data tests were analysed using the non-parametric ANOVA test.

\subsection{Free Radical Damage in the Test Tube, in Vitro Model:}

We reported earlier that protein cross-linking as a model for detection of free radical activity and activation of
Table 2. Groups tested (8 teeth per groups).

\begin{tabular}{cc}
\hline Group A & $\begin{array}{c}\text { 37\% of phosphoric acid + primer + bonding } \\
\text { immediately (negative control) }\end{array}$ \\
Group B & $\begin{array}{c}\text { Self-etching primer + bonding immediately } \\
\text { (positive control) }\end{array}$ \\
Group C & Gel1 + primer + bonding immediately \\
Group D & Gel2 + primer + bonding immediately \\
Group E & Gel3 + primer + bonding immediately \\
Group F & Gel4 + primer + bonding immediately \\
Group K & Gel5 + primer + bonding immediately \\
Group L & Gel6 + primer + bonding immediately \\
Group M & Gel7 + primer + bonding immediately \\
\hline
\end{tabular}

"molecular defense forces". Bovine serum albumin (BSA), a completely water-soluble protein, was polymerized by hydroxyl radicals generated by the Fenton reaction system of $\mathrm{Fe}^{2+} /$ EDTA $/ \mathrm{H}_{2} \mathrm{O}_{2} /$ ascorbate [12]. As a result, the protein loses its water-solubility and the polymerized product precipitates. The decrease in the concentration of the water-soluble protein can easily be detected. The in vitro incubation mixtures contained reagents, added in the sequence as follows, at the final concentrations: bovine serum albumin $(0.8 \mathrm{mg} / \mathrm{ml})$, phosphate buffer, pH $7.4(10 \mathrm{mM})$, water to reach $2.5 \mathrm{ml}$ total volume, antioxidant tested to reach required concentration as shown in results, EDTA $(0-/ 4.8 \mathrm{mM}), \mathrm{Fe}\left(\mathrm{NH}_{4}\right)_{2}$ $\left(\mathrm{SO}_{4}\right)_{2}(0-/ 4 \mathrm{mM})$, ascorbate $(4 \mathrm{mM})$ and $\mathrm{H}_{2} \mathrm{O}_{2}(0.2 \%)$. To chelate iron completely, 1.2 molar excess of EDTA was always use [13]. The reaction mixture was incubated for $20 \mathrm{~min}$ at ambient temperature. The supernatant was precipitated with an equal volume of trichloroacetic acid $(10 \%)$ at $0^{\circ} \mathrm{C}$. The precipitate thus obtained was re-dissolved in $1 \mathrm{ml}$ of $\mathrm{Na}_{2} \mathrm{CO}_{3}(10 \%)$ in $\mathrm{NaOH}(0.5 \mathrm{M})$ and the final volume made up to $2.5 \mathrm{ml}$ by water. An aliquot of the solution was used for protein determination [14]. The yield of $\mathrm{OH}$. radicals generated in the incubations was determined on the basis of degradation of deoxyribose [14]. Bityrosine formation was monitored by measuring fluorescence at $325 \mathrm{~nm}$ (excitation) and $415 \mathrm{~nm}$ (emission) according spectrophotometer [15].

\section{RESULTS}

\subsection{The Characterization of NSAID-Chitosan Gels (Gel 1-7)}

The SEM images were obtained to characterize the microstructure of the freeze-dried naproxen composite gels and are presented in Figure 1. It could be seen that the gels displayed a homogeneously pore structure. It was thought that the micro-porous structure of the gels could 

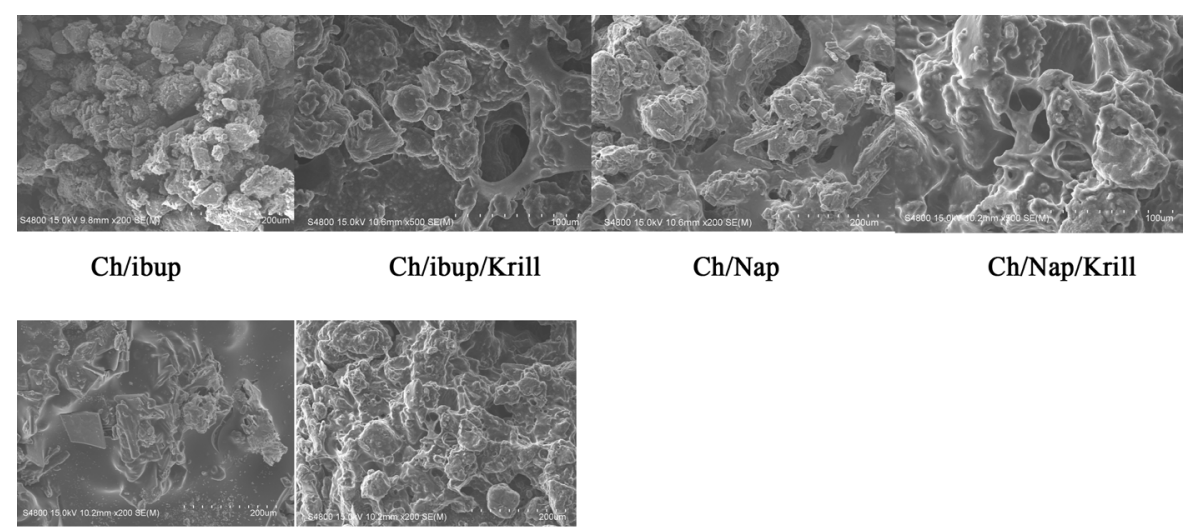

$\mathrm{Ch} /$ Aspirin

$\mathrm{Ch} /$ Aspirin/Krill

Figure 1. SEM photographs of interior morphology of the selected gels under investigation for (a) Ch/Ib, (b) Ch/Ibup/Krill, (c) Ch/Nap, (d) Ch/Aspirin/Krill, (e) Gel-6, (f) Gel-7.

lead to high internal surface areas with low diffusional resistance in the gels. The surfaces of the gels were also presented (Figure 1). The "skin" of the gels can be seen, and the collapse of the surface pores may be due to freeze-drying process.

\subsection{Studies of Equilibrium Swelling in NSAIDs-Chitosan Gels (Gel 1-Gel 7)}

The hydrogels remain in the cylindrical form after swelling. Compared with dry state hydrogels, the swollen state hydrogel volume displays significant increases and are summarized in Figure 2.

Equilibrium swelling ratio (SR) of hydrogels exerts an influence on their release rates. The reduction in equilibrium swelling capacity is due to the formation of a tight network structure in high content. Environmental $\mathrm{pH}$ value has a large effect on the swelling behavior of these gels. From Figure 2, it is clear that the SR value increases with the increase of $\mathrm{pH}$. Such $\mathrm{pH}$ dependent properties of the hydrogels come from the polyelectrolyte nature of chitosan segments in the hydrogel network. Namely, when the $\mathrm{pH}$ value of the buffer solution $(\mathrm{pH}$ 9.0) was far higher than the isoelectric point (PI) of GEL (PI 4.0 - 5.0), the carboxyl groups were de-protonized to carry negative charges, which made molecular chains repulsed to each other. The network became looser and it was easy for the water molecules to diffuse into the cross-linked network. According to above results, we believed that the naproxen results release mechanism could result from the superposition of various effects, such as swelling property of hydrogels, the solubility of the drug and erosion property of matrix; it is not necessarily based on a single factor.

\subsection{Shear Bond Strengths}

Figure 3 gives the shear bond strength values (MPa)

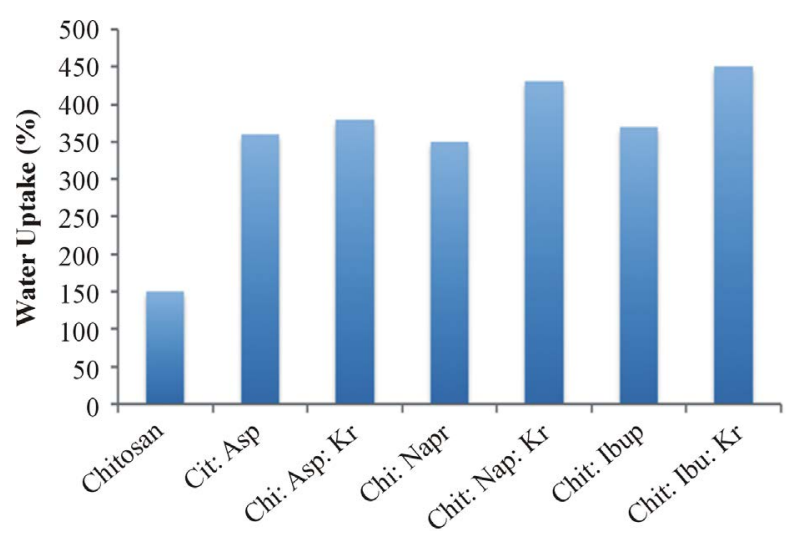

Figure 2. Water uptake degree of the gels Gel $1-$ Gel $7(n=6$, $\mathrm{p}<0.05)$.

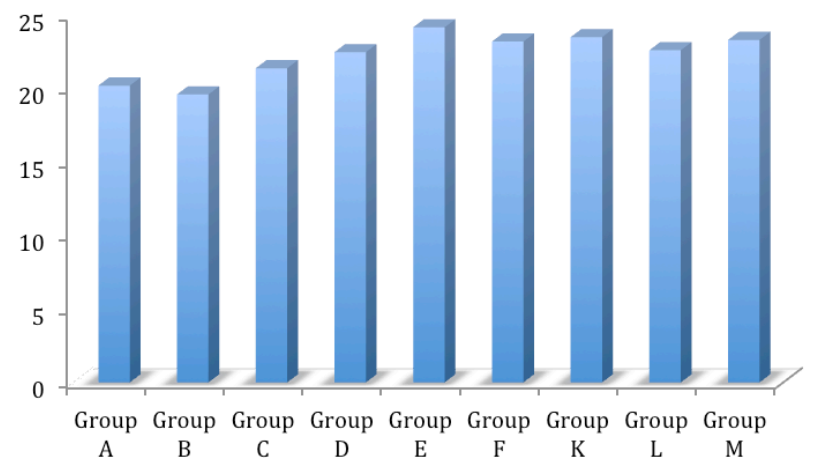

Figure 3. Shear bond strength of hydrogels after 24 hours of bonding to dentin.

after 24 hours.

Mean shear bond strength values and difference between the groups are summarized in Figure 3 for bonding to dentin after 24 hours. In general there was an increase in bond strength of the dentin treated with the antioxidant containing hydrogels compared to the bond strength of the conventionally bonded teeth. An increase 
in the shear bond strength was also previously reported [16] for chitosan-H, chitosan-asprin, chitosan-asprin-krill, chitosan-naproxen, chitosan-naproxen-krill, chitosanibuprofen and chitosan: ibuprofen: krill Interestingly the increase in bond strength was also observed in the groups of hydrogen peroxide exposed samples suggesting that there additional benefits associated with chitosan: antioxidant system are in need of further investigations [16].

The results of this study suggest that the optimum results for the strengthening of dentin can be achieved throughout the immediate treatment with antioxidant: chitosan with the increase of dentin bond strength. Also, impressively an almost immediately after the corresponding gel treatment and proceeding with bonding procedures is recommended with the significant increase in bond strength. The results of this study suggest that the optimum results for the increased enamel dentin bond strength can be achieved through out the immediate treatment with gels. The additional advantage of the system may suggest that, antioxidant release from chitosan gel depends on the physical network structure (open cell like structure) as well as $\mathrm{pH}$ properties and flexibilities of the material. Antioxidant release occurs through the pores of the low polymer concentration while chitosan concentration increment resulted in more cross-linking of the network structure; consequently slower antioxidant release from the gel base was achieved and therefor weaker adhesive properties of the materials such as Gel1 in case of groups [17].

It was shown by others and us earlier, that the swelling properties and antioxidant release from gels were increased under acidic conditions due to the protonation of the primary amino group on chitosan [18]. Chain relaxation due to protonation of amino groups leads to a faster hydrogen bond dissociation and efficient solvent diffusion. Thus, the appreciable increase in water uptake at lower $\mathrm{pH}$ values can be attributed to the high porosity of the gels, which seems to govern the diffusion of the solvent in the gel matrix, and thus, the release of the antioxidant from the gel [19]. The additional benefit of using chitosan: antioxidant system as a bonding/pre-bonding to enamel and dentin system lies in its ability to show favorable immediate results in terms of bonding effectiveness as well as the durability of resin-dentin bonds for a prolonged time (up to 6 months) [19]. It is well documented that the hydrostatic pulpal pressure, the dentinal fluid flow and the increased dentinal wetness in vital dentin can affect the intimate interaction of certain enamel and dentin adhesives with dentinal tissue. Therefor the newly developed chitosan: antioxidant systems might at least be able to address the shortfalls in the current perspectives for improving bond durability through understanding factors affecting the long-term bonding performance of modern adhesives and addresses the current perspectives for improving bond durability.

\subsection{In Vitro Release of Naproxen from Naproxen-Chitosan Gels (Gels 4-5)}

The in vitro release of naproxen from chitosan gels was carried out using USP dissolution apparatus type I. As the regression analysis of the obtained results for two kinetic models including zero order and Higushi's model showed that Higushi's model gave the highest value of $\mathrm{r}^{2}$ with significant difference $(\mathrm{p}<0.05)$. Higushi's model, where the cumulative amount of the released drug per unit area is proportional to the square root of time, is more suitable model to describe the release kinetics of from the gel preparations examined in the present study. The release of naproxen from chitosan gel 5\% was studied and gels containing corresponding antioxidant and BSA, as shown in Figure 4. The principal mechanism of such interactions is the formation of hydrogen bonds involving amino group and carboxyl group of naproxen. Also its becomes apparent that the influence of chemical structures of antioxidants such as krill oil have significantly improved the release of naproxen from the hydrogels. The mechanism of this interaction is currently under investigations in our laboratories.

\subsection{In Vitro Release of Ibuprofen from Ibuprofen-Chitosan Gels (Gels 6-7)}

The in vitro release of ibuprofen from chitosan gels was carried out using USP dissolution apparatus type I. As the regression analysis of the obtained results for two kinetic models including zero order and Higushi's model showed that Higushi's model gave the highest value of $\mathrm{r}^{2}$ with significant difference $(\mathrm{p}<0.05)$. Higushi's model, where the cumulative amount of the released drug per unit area is proportional to the square root of time, is more suitable model to describe the release kinetics of ibuprofen from the gel preparations examined in the present study. The release of ibuprofen from chitosan gel

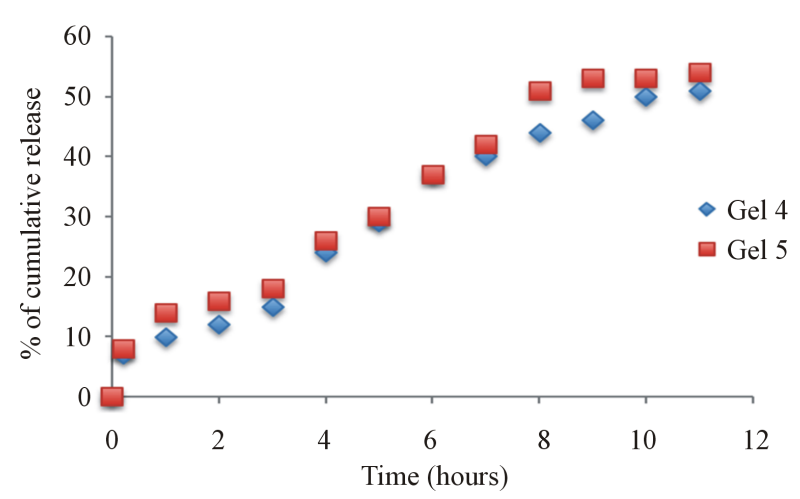

Figure 4. The effect additive such as Krill oil and chitosan release from 5\% w/w chitosan gels (Gel 4 and Gel 5) in phosphate buffer $\mathrm{pH} 6.8$. 
$5 \%$ was studied and gels containing corresponding antioxidant and BSA, as shown in Figure 5. The principal mechanism of such interactions is the formation of hydrogen bonds involving amino group and carboxyl group of ibuprofen. Also its becomes apparent that the influence of chemical structures of synergetic antioxidant such as krill oil have significantly improved the release of naproxen from the hydrogels. The mechanism of this interaction is currently under investigations in our laboratories.

\subsection{In Vitro Release of Aspirin from Aspirin-Chitosan Gels (Gel 2-3)}

The in vitro release of aspirin from chitosan gels was carried out using USP dissolution apparatus type I. As the regression analysis of the obtained results for two kinetic models including zero order and Higushi's model showed that Higushi's model gave the highest value of $r^{2}$ with significant difference $(\mathrm{p}<0.05)$. Higushi's model, where the cumulative amount of the released drug per unit area is proportional to the square root of time, is more suitable model to describe the release kinetics of from the gel preparations examined in the present study. The release of aspirin from chitosan gel 5\% was studied and gels containing corresponding antioxidant and BSA, as shown in Figure 6. The principal mechanism of such interactions is the formation of hydrogen bonds involving amino group and carboxyl group of naproxen. Also its becomes apparent that the influence of chemical structures of antioxidants such as krill oil have significantly improved the release of naproxen from the hydrogels. The mechanism of this interaction is currently under investigations in our laboratories.

\subsection{NSAIDs and Chitosan Looking at the Antioxidant Strength and Extended Stability, Could Macroencapsulation Play a Functional Role}

Nutraceutical carriers which can enclose bioactive com-

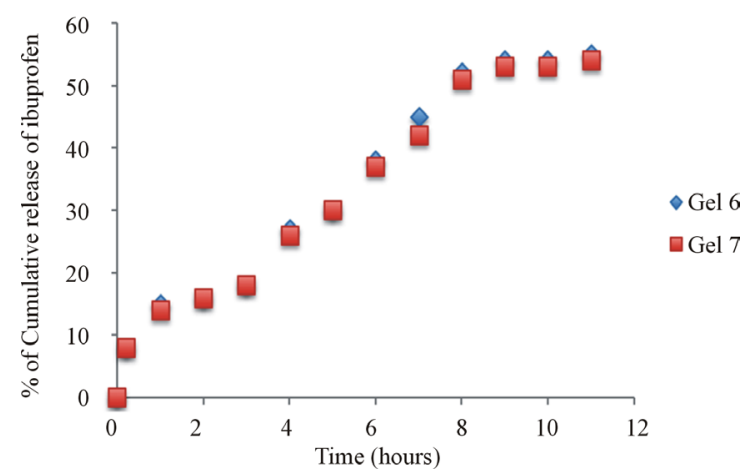

Figure 5. The effect additive such as Krill oil and chitosan release of ibuprofen.

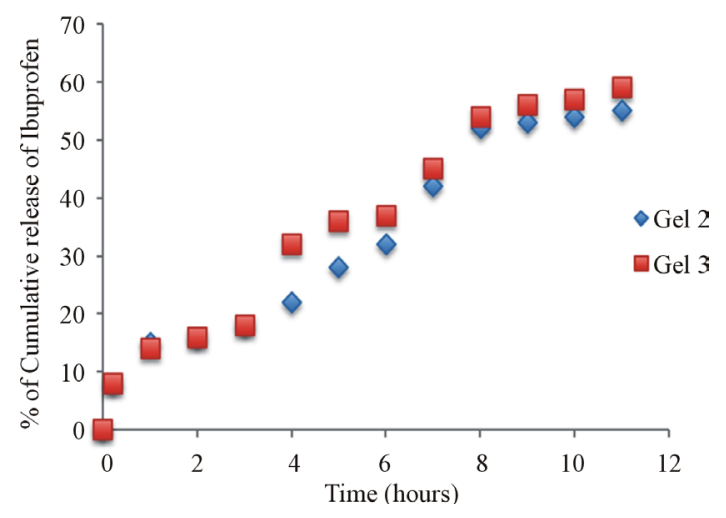

Figure 6. The effect additive such as Krill oil and chitosan release from $5 \% \mathrm{w} / \mathrm{w}$ chitosan gels (Gels 2 and 3 ) in phosphate buffer $\mathrm{pH} 6.8$.

pounds such as protein and vitamin with health benefits provide an excellent opportunity to improve public health $[19,20]$. The nutraceutical carriers are required to have the ability to protect the active compound against chemical degradation by the surrounding dispersion medium and control the release rate of the incorporated compound. As the material of the carrier, it is also required to be non-toxic, biocompatible and biodegradable $[21,22]$. Chitosan, a linear abundant polysaccharide, is selected as the wall material of the delivery system. Due to its biodegradable, biocompatible, mucoadhesive and non-toxic nature [23-25], it has been widely used in numerous drug delivery systems. Compared to other delivery systems, chitosan nanoparticles have a special feature. They can adhere to the mucosal surface and transiently open the tight junction between epithelial cells. Some reports have indicated that chitosan can increase membrane permeability, both in vitro [26-28] and in vivo [29]. Microencapsulation of antioxidants have been important area of research for several year in order to preserve the beneficial effects of antioxidants [30-32]. In this work, the gelation method was used to prepare the Chitosan: Krill oil, Asprin: Chitosan, Ibuprofen: Chitosan, Naproxen: Chitosan microencapsulation. The stability of antioxidant-loaded chitosan complexes has been measured during storage and no significant decomposition observed after 6 month storage at room temperature $\left(24^{\circ} \mathrm{C}\right)$.

\subsection{Stability of Antioxidants Such as (Krill Oil, Aspirin, Naproxen and Ibuprofen) in the Chitosan Hydrogels during Storage}

Stability of various conventional antioxidants in the newly designed drug delivery system during storage is an important factor to determine whether chitosan-coated nano-size delivery vehicle can protect various conventional antioxidants (Figures 7-12). So the stability of the microencapsulated antioxidants has been measured by 


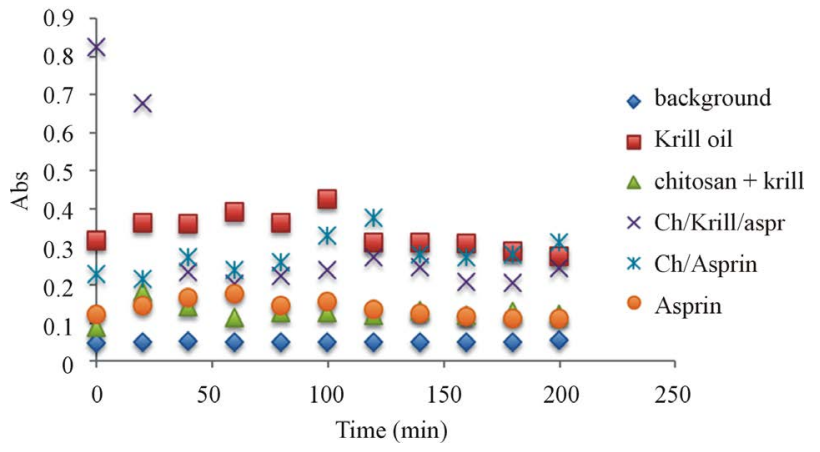

Figure 7. Antioxidant capacity measured at $450 \mathrm{~nm}$ using the previously described spectrophotometric assay to assess the hydrogels and corresponding ingredients antioxidant capacity after 24 hours under storage under ambient temperature condition. Antioxidant capacity was measured during the first 2 hours of exposure.

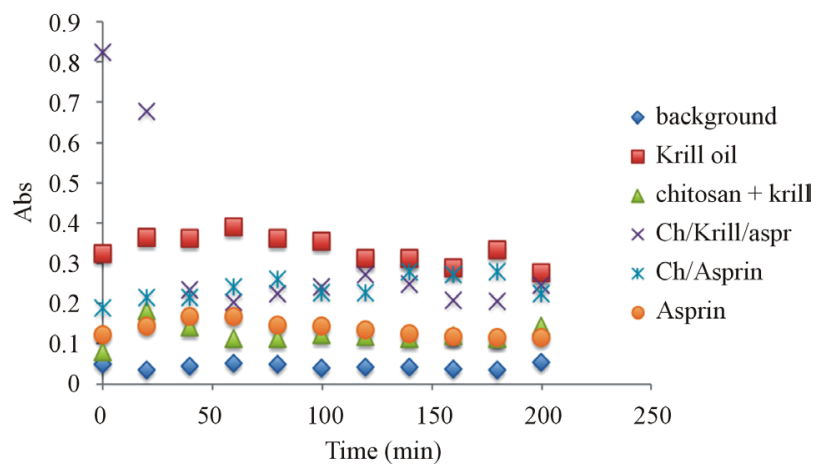

Figure 8. Antioxidant capacity measured at $450 \mathrm{~nm}$ using the previously described spectrophotometric assay to assess the hydrogels and corresponding ingredients antioxidant capacity after 6 month under storage under ambient temperature condition. Antioxidant capacity was measured during the first 2 hours of exposure.

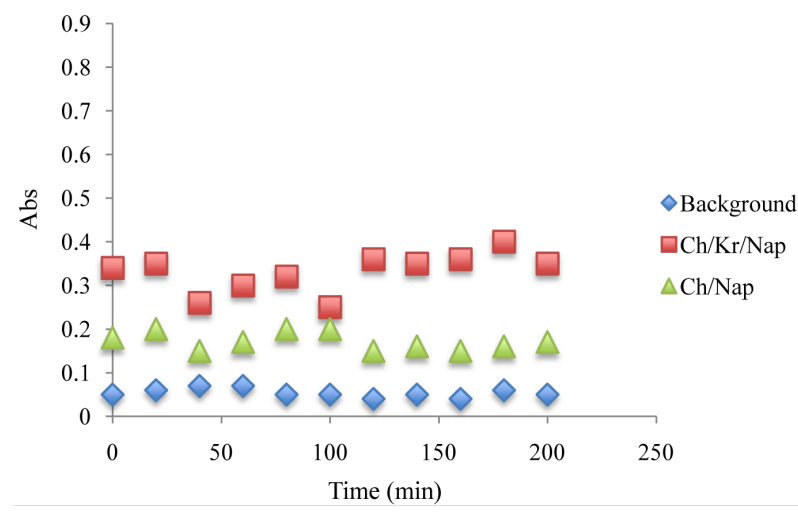

Figure 9. Antioxidant capacity measured at $450 \mathrm{~nm}$ using the previously described spectrophotometric assay to assess the hydrogels and corresponding ingredients antioxidant capacity after 24 hours under storage under ambient temperature condition. Antioxidant capacity was measured during the first 2 hours of exposure.

UV absorbance. Stabilities of microencapsulated anti-

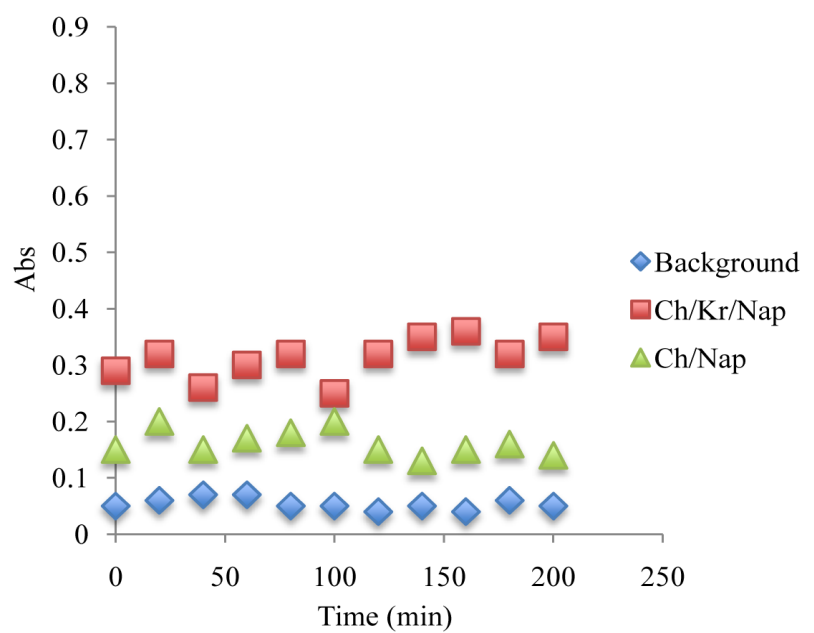

Figure 10. Antioxidant capacity measured at $450 \mathrm{~nm}$ using the previously described spectrophotometric assay to assess the hydrogels and corresponding ingredients antioxidant capacity after 6 month under storage under ambient temperature condition. Antioxidant capacity was measured during the first 2 hours of exposure.

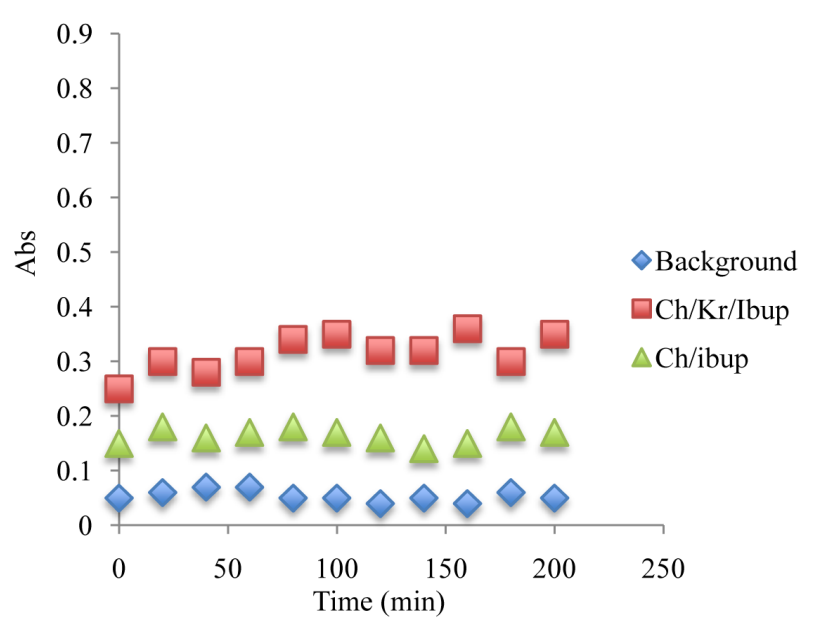

Figure 11. Antioxidant capacity measured at $450 \mathrm{~nm}$ using the previously described spectrophotometric assay to assess the hydrogels and corresponding ingredients antioxidant capacity after 24 hours under storage under ambient temperature condition. Antioxidant capacity was measured during the first 2 hours of exposure.

oxidants have been compared and after 6 month of storage at $24^{\circ} \mathrm{C}$, the stability of antioxidant-molecular carrier vehicle was over $95 \%$. This indicated that the antioxidant had been protected by the molecular carrier such as chitosan. Important to note that performance of the antioxidant was enhanced by the presence of the chitosan, which is a very interesting point in itself as the synergism in increased stability and lower concentration of the active antioxidant wit the same or even higher antioxidant capacity can lead to a development of broad range to novel functional drug delivery systems. 


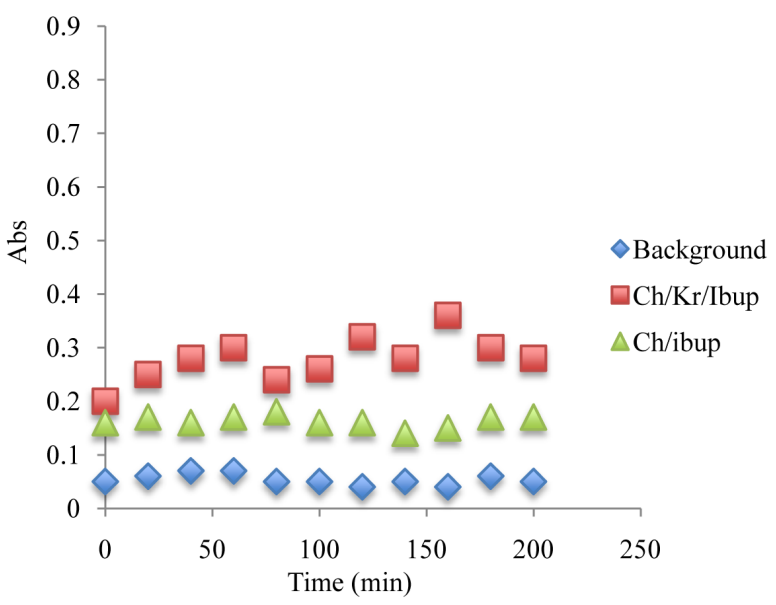

Figure 12. Antioxidant capacity measured at $450 \mathrm{~nm}$ using the previously described spectrophotometric assay to assess the hydrogels and corresponding ingredients antioxidant capacity after 6 month under storage under ambient temperature condition. Antioxidant capacity was measured during the first 2 hours of exposure.

\subsection{Further Developments in the "Build in Free Radical Detector" inside the System and Solubility of BSA and Simultaneous Corrective Mechanism for Newly Born Free Radical Quenching Molecular Controller}

In the present work we adopted the method of Zs.-Nagy and Nagy [33] for recording changes in the water solubility of BSA exposed to the chemical source of hydroxyl free radicals to characterize the antioxidant efficiency of Krill oil, aspirin, ibuprofen and naproxen and complex with chitosan in a non-lipid protein system. We used the Fenton reaction system of $\mathrm{Fe}^{2+} /$ EDTA $/ \mathrm{H}_{2} \mathrm{O}_{2} /$ ascorbate [34] which gave a defined flow of hydroxyl radicals. We used deoxyribose as a detection molecule to determine the yield of hydroxyl radicals in the Fenton reaction system of $\mathrm{Fe}^{2+} /$ EDTA $/ \mathrm{H}_{2} \mathrm{O}_{2}$ /ascorbate. At $2 \mathrm{mM}$ [Fe-ED$\mathrm{TA}]^{2+}, \mathrm{OH}$ radicals were generated almost linearly up to 10 min and then the radical production ceased; after 30 min $\mathrm{OH}$ generation was no longer observed. The total integral yield of hydroxyl radicals was found to be 15 $\mathrm{nmol} / \mathrm{ml}$. BSA, a completely water soluble protein, exposed to the above Fenton reaction system, was losing its water solubility depending on the concentration of the chelated iron, as shown in Figure 13. The in-solubilization was slow up to about $0.8 \mathrm{mM}$ iron; a loss of $50 \%$ solubility was recorded around $1.3 \mathrm{mM}[\text { Fe-EDTA }]^{2+}$, and practically complete precipitation occurred above 2.0 $\mathrm{mM}$ iron. Total molar amount of BSA precipitated in relation to $\mathrm{OH}$ radical total yield gave factor 1.25 . Control experiments showed that omission of either chelated iron or $\mathrm{H}_{2} \mathrm{O}_{2}+$ ascorbate from the reaction mixture gave no decrease in protein solubility (data not shown). Characteristic fluorescence of 325 (excitation)/415 (emission)

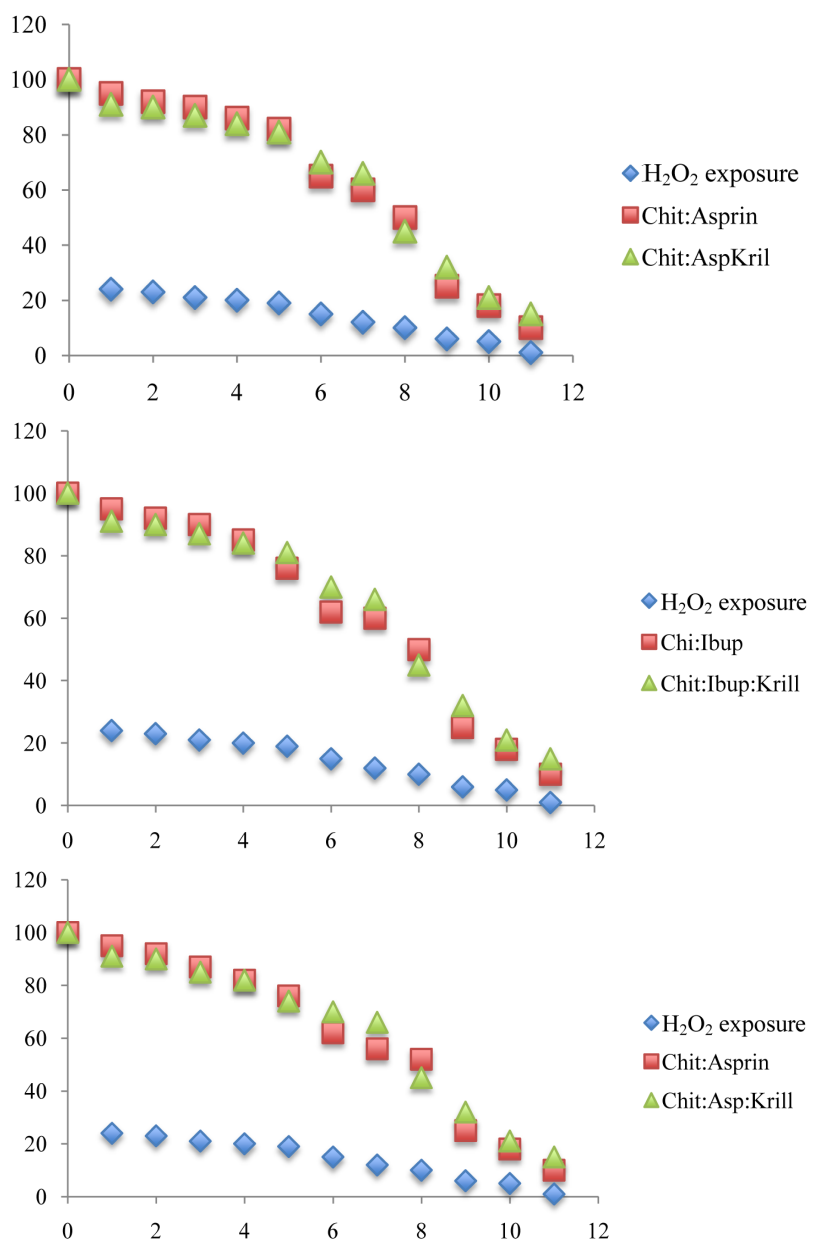

Figure 13. Affects of the various antioxidant on the solubility of BSA protein in the drug delivery system.

was significantly increased in incubations of BSA with the Fenton system fortified with $0.75 \mathrm{mM}$ ferrous chelate, indicating the presence of bityrosine covalent bridges. The specific yield of bityrosine fluorescence was found to be strongly dependent on the initial concentration of BSA, which suggests an intermolecular character of bityrosine cross-links: maximal yield was observed at 0.33 $\mathrm{mg} / \mathrm{ml}$; both at higher and lower protein concentrations the specific fluorescence gain was lower $[35,36]$. On balance then, the results obtained in this study strongly indicated that the insolubilization of BSA induced by the Fenton system of $\mathrm{Fe}^{2+} /$ EDTA/ $/ \mathrm{H}_{2} \mathrm{O}_{2} /$ ascorbate was caused by free $\mathrm{OH}$ radical mediated polymerization giving rise to true covalent cross-links. The model system was found suitable for convenient testing of $\mathrm{OH}$ radical scavenging ability of new antioxidants in a non-lipid environment.

We considered worthwhile to study the antioxidant properties of krill oil, aspirin, naproxen and ibuprofen in a non-lipid environment of a pure protein. Therefore we adopted the method for recording changes in water solubility of the model protein bovine serum albumin (BSA) 
exposed to free radicals generated by an inorganic chemical system. In the present study we used the Fenton reaction system of $\mathrm{Fe}^{2+} / \mathrm{EDTA} / \mathrm{H}_{2} \mathrm{O}_{2}$ /ascorbate as a source of free radicals to prove the ability of NSAIDs as well as complexed form of Chitosan: NSADs to protect BSA against free radical mediated cross-linking, in comparison with an efficient hydroxyl radical scavenger such as trolox.

\section{CONCLUSION}

We developed and tested functional dual action restorative materials capable of being "bio-active" and longlasting, we designed and evaluated novel chitosan hydrogels containing krill oil (antioxidant containing material), naproxen, ibuprofen (non steroidal anti-inflammatory medication), aspirin (pain relieve medication and free radical scavengers) and combinations thereof (chitosan$\mathrm{H}$-krill oil, chitosan-H-krill oil-aspirin and chitosan-Hnaproxen, chitosan-H-naproxen-krill oil, chitosan-H-krill oil-ibuprofen and chitosan-H-ibuprofen) as functional additive prototypes for further development of "dual function restorative materials". We quantified the effects of functional designer biomaterials on the dentin bond strength of a composite and evaluated the capability of newly designed hydrogels to play an integral role of "build in" free radical defense mechanism by using BSA solubility as a "molecular prototype" of the site of free radical attack in vitro. The added benefits of the chitosan treated hydrogels involved positive influence on the aspirin, ibuprofen and naproxen release, increased dentin bond strength as well as demonstrated in vitro "build in" free radical defense mechanism, therefore acting as a "proof of concept" for the functional multi-dimensional restorative materials with the build in free radical defense mechanism.

\section{REFERENCES}

[1] Lefer, L.A. (1966) Psychoanalytic view of a dental phenomenon. Contemporary Psychoanalysis, 2, 135.

[2] Fine, E.W. (1971) Psychological factors associated with non-organic TMJ dysfunction syndrome. British Dental Journal, 131, 402-427. http://dx.doi.org/10.1038/sj.bdj.4802760

[3] Feinmann, C. and Harris, M. (1984) Psychogenic facial pain. 1. The clinical presentation. British Dental Journal, 156, 165-168. http://dx.doi.org/10.1038/sj.bdj.4805298

[4] Feinmann, C. and Harris, M. (1984) Psychogenic facial pain. Management and prognosis. British Dental Journal, 156, 205-208.

[5] Aghabeigi, B., Feinmann, C., Glover, V., et al. (1993) Tyramine conjugation deficit in patients with chronic idiopathic temporomandibular joint and orofacial pain. Pain, 54, 159-163.

http://dx.doi.org/10.1016/0304-3959(93)90204-3
[6] Fundueanu, G., Constantin, M. and Ascenzi, P. (2008) Preparation and characterization of $\mathrm{pH}$ - and temperaturesensitive pullulan microspheres for controlled release of drugs. Biomaterials, 29, 2767-2775.

http://dx.doi.org/10.1016/j.biomaterials.2008.03.025

[7] Huynh, D.P., Nguyen, M.K., Pi, B.S., Kim, M.S., Chae, S.Y., Lee, K.C., et al. (2008) Functionalized injectable hydrogels for controlled insulin delivery. Biomaterials, 29, 2527-2534.

http://dx.doi.org/10.1016/j.biomaterials.2008.02.016

[8] Wang, Y.-C., Liu, X.-Q., Sun, T.-M., Xiong, M.-H. and Wang, J. (2008) Functionalized micelles from block copolymer of polyphosphoester and poly(3-caprolactone) for receptormediated drug delivery. Journal of Controlled Release, 128, 32-40. http://dx.doi.org/10.1016/j.jconrel.2008.01.021

[9] Nakamura, K., Maitani, Y., Lowman, A.M., Takayama, K., Peppas, N.A. and Nagai, T. (1999) Uptake and release of budesonide from mucoadhesive, $\mathrm{pH}$-sensitive copolymers and their application to nasal delivery. Journal of Controlled Release, 61, 329-335. http://dx.doi.org/10.1016/S0168-3659(99)00150-9

[10] Mok, H., Park, J.W. and Park, T.G. (2008) Enhanced intracellular delivery of quantum dot and adenovirus nanoparticles triggered by acidic $\mathrm{pH}$ via surface charge reversal. Bioconjugate Chemistry, 19, 797-801. http://dx.doi.org/10.1021/bc700464m

[11] He, C., Kim, S.W. and Lee, D.S. (2008) In situ gelling stimuli-sensitive block copolymer hydrogels for drug delivery. Journal of Controlled Release, 127, 189-207. http://dx.doi.org/10.1016/j.jconrel.2008.01.005

[12] Nguyen, D.N., Raghavan, S.S., Tashima, L.M., Lin, E.C., Fredette, S.J., Langer, R.S., et al. (2008) Enhancement of poly(orthoester) microspheres for DNA vaccine delivery by blending with poly(ethylenimine). Biomaterials, 29, 2783-2793.

http://dx.doi.org/10.1016/j.biomaterials.2008.03.011

[13] Bae, Y. and Kataoka, K. (2006) Significant enhancement of antitumor activity and bioavailability of intracellular $\mathrm{pH}$-sensitive polymeric micelles by folate conjugation. Journal of Controlled Release, 116, e49-e50. http://dx.doi.org/10.1016/j.jconrel.2006.09.044

[14] Kim, J., Conway, A. and Chauhan, A. (2008) Extended delivery of ophthalmic drugs by silicone hydrogel contact lenses. Biomaterials, 29, 2259-2269.

http://dx.doi.org/10.1016/j.biomaterials.2008.01.030

[15] Tang, Y. and Singh, J. (2008) Controlled delivery of aspirin: Effect of aspirin on polymer degradation and in vitro release from PLGA based phase sensitive systems. International Journal of Pharmaceutics, 357, 119-125. http://dx.doi.org/10.1016/j.ijpharm.2008.01.053

[16] Watanabe, M., Kawano, K., Toma, K., Hattori, Y. and Maitani, Y. (2008) In vivo antitumor activity of camptothecin incorporated in liposomes formulated with an artificial lipid and human serum albumin. Journal of Controlled Release, 127, 231-238. http://dx.doi.org/10.1016/j.jconrel.2008.02.005

[17] Tamilvanan, S., Venkateshan, N. and Ludwig, A. (2008) The potential of lipid- and polymerbased drug delivery 
carriers for eradicating biofilm consortia on devicerelated nosocomial infections. Journal of Controlled Release, 128, 2-22. http://dx.doi.org/10.1016/j.jconrel.2008.01.006

[18] Lee, M.-H., Lin, H.-Y., Chen, H.-C. and Thomas, J.L. (2008) Ultrasound mediates the release of curcumin from microemulsions. Langmuir, 24, 1707-1713. http://dx.doi.org/10.1021/la7022874

[19] Connal, L.A., Li, Q., Quinn, J.F., Tjipto, E., Caruso, F. and Qiao, G.G. (2008) pH-responsive poly (acrylic acid) core cross-linked star polymers: Morphology transitions in solution and multilayer thin films. Macromolecules, 41, 2620-2626. http://dx.doi.org/10.1021/ma7019557

[20] Kurkuri, M.D., Nussio, M.R., Deslandes, A. and Voelcker, N.H. (2008) Thermosensitive copolymer coatings with enhanced wettability switching. Langmuir, 24, 42384244. http://dx.doi.org/10.1021/la703668s

[21] Chen, S., Li, Y., Guo, C., Wang, J., Ma, J., Liang, X., et al. (2007) Temperature-responsive magnetite/PEO-PPOPEO block copolymer nanoparticles for controlled drug targeting delivery. Langmuir, 23, 12669-12676. http://dx.doi.org/10.1021/la702049d

[22] Shah, N.M., Pool, M.D. and Metters, A.T. (2006) Influence of network structure on the degradation of photocross-linked PLA-b-PEG-b-PLA hydrogels. Biomacromolecules, 7, 3171-3177. http://dx.doi.org/10.1021/bm060339z

[23] Nolkrantz, K., Farre, C., Brederlau, A., Karlsson, R.I.D., Brennan, C., Eriksson, P.S., et al. (2001) Electroporation of single cells and tissues with an electrolyte-filled capillary. Analytical Chemistry, 73, 4469-4477. http://dx.doi.org/10.1021/ac010403x

[24] Nguyen, K.T. and West, J.L. (2002) Photopolymerizable hydrogels for tissue engineering applications. Biomaterials, 23, 4307-4314. http://dx.doi.org/10.1016/S0142-9612(02)00175-8

[25] Roy, I. and Gupta, M.N. (2003) Smart polymeric materials: Emerging biochemical applications. Chemistry \& Biology, 10, 1161-1171. http://dx.doi.org/10.1016/j.chembiol.2003.12.004

[26] Katime, I., Novoa, R., de Apodaca, E.D. and Rodríguez, E. (2004) Release of theophylline and aminophylline from acrylic acid/n-alkyl methacrylate hydrogels. Journal of Polymer Science Part A: Polymer Chemistry, 42, 27562765. http://dx.doi.org/10.1002/pola.20112

[27] Alam, H.B., Burris, D., DaCorta, J.A. and Rhee, P. (2005) Hemorrhage control in the battlefield: Role of new hemostatic agents. Military Medicine, 170, 63-69.
[28] Amiji, M.M. (1995) Permeability and blood compatibility properties of chitosan-poly (ethylene oxide) blend membranes for haemodialysis. Biomaterials, 16, 593-599. http://dx.doi.org/10.1016/0142-9612(95)93856-9

[29] Barrera, D.A., Zylstra, E., Lansbury, P.T. and Langer, R. (1993) Synthesis and RGD peptide modification of a new biodegradable copolymer: Poly(lactic acid-co-lysin). Journal of the American Chemical Society, 115, 11010-11011. http://dx.doi.org/10.1021/ja00076a077

[30] Baumann, H. and Faust, V. (2001) Concepts for improved regioselective placement of O-sulfo, N-sulfo, Nacetyl, and $\mathrm{N}$-carboxymethyl groups in chitosan derivatives. Carbohydrate Research, 331, 43-57. http://dx.doi.org/10.1016/S0008-6215(01)00009-X

[31] Beena, M.S., Chandy, T. and Sharma, C.P. (1995) Heparin immobilized chitosan-poly ethylene glycol interpenetrating network: Antithrombogenicity. Artificial Cells, Blood Substitutes and Biotechnology, 23, 175-192. http://dx.doi.org/10.3109/10731199509117937

[32] Bordenave, L.C., Lbaquey, R., Bareille, F., Lefebvre, C., Lauroua, V., Guerin, F., Rouais, N., More, C., Vergnes, C. and Anderson, J.M. (1995) Endothelial-cell compatibility testing of 3 different pelletanes. Journal of Biomedical Materials Research, 27, 1367-1381. http://dx.doi.org/10.1002/jbm.820271104

[33] Brown, M., Daya, M. and Worley, J. (2009) Experience with chitosan dressings in a civilian EMS system. Journal of Emergency Medicine, 37, 1-7. http://dx.doi.org/10.1016/j.jemermed.2007.05.043

[34] Carreno-Gomez, B. and Duncan, R. (1997) Evaluation of the properties of soluble chitosan and chitosan microspheres. International Journal of Pharmaceutics, 148, 231-240. http://dx.doi.org/10.1016/S0378-5173(96)04847-8

[35] Cenni, E., Ciapetti, G., Cervellati, M., Cavedagna, D., Falsone, G., Gamberini, S. and Pizzoferrato, A. (1996) Activation of the plasma coagulation system induced by some biomaterials. Journal of Biomedical Materials Research, 31, 145-148. http://dx.doi.org/10.1002/(SICI)1097-4636(199605)31:1< 145::AID-JBM16>3.0.CO;2-M

[36] Cerchiara, T., BLuppi, B., Bigucci, F., Petrachi, M., Orienti, I. and Zecchi, V. (2003) Controlled release of vancomycin from freeze-dried chitosan salts coated with different fatty acids by spray-drying. Journal of Microencapsulation, 20, 473-478. http://dx.doi.org/10.1080/0265204031000094329 